\title{
BMJ Global Health A mixed-methods study of maternal health care utilisation in six referral hospitals in four sub-Saharan African countries before and during the COVID-19 pandemic
}

Aduragbemi Banke-Thomas (D) , ${ }^{1,2}$ Aline Semaan, ${ }^{3}$ Dinah Amongin (D) ,,5 Ochuwa Babah, ${ }^{6}$ Nafissatou Dioubate, ${ }^{7}$ Amani Kikula, ${ }^{8}$ Sarah Nakubulwa, ${ }^{4,9}$ Olubunmi Ogein, ${ }^{6}$ Moses Adroma, ${ }^{4}$ William Anzo Adiga, ${ }^{10}$ Abdourahmane Diallo, ${ }^{11}$ Lamine Diallo, ${ }^{12}$ Mamadou Cellou Diallo, ${ }^{11}$ Cécé Maomou, ${ }^{12}$ Nathanael Mtinangi, ${ }^{13}$ Telly Sy, ${ }^{11}$ Therese Delvaux, ${ }^{3}$ Bosede Bukola Afolabi (D) , ${ }^{6}$ Alexandre Delamou (D) , ${ }^{7,14}$ Annettee Nakimuli, ${ }^{4}$ Andrea Barnabas Pembe, ${ }^{8}$ Lenka Benova ${ }^{3}$

To cite: Banke-Thomas $A$, Semaan A, Amongin D, et al. A mixed-methods study of maternal health care utilisation in six referral hospitals in four sub-Saharan African countries before and during the COVID-19 pandemic. BMJ Global Health 2022;7:e008064. doi:10.1136/ bmjgh-2021-008064

Handling editor Seye Abimbola

- Additional supplemental material is published online only. To view, please visit the journal online (http://dx.doi.org/10. 1136/bmjgh-2021-008064)

Received 20 November 2021 Accepted 20 January 2022

Check for updates

(c) Author(s) (or their employer(s)) 2022. Re-use permitted under CC BY-NC. No commercial re-use. See rights and permissions. Published by BMJ.

For numbered affiliations see end of article.

Correspondence to Dr Aduragbemi Banke-Thomas; a.bankethomas@gre.ac.uk

\section{ABSTRACT}

Introduction In sub-Saharan Africa, referral hospitals are important sources of key maternal health services, especially during a crisis such as the COVID-19 pandemic. This study prospectively assessed the effect of the COVID-19 pandemic on maternal health service utilisation in six large referral hospitals in Guinea, Nigeria, Tanzania and Uganda during the first year of the pandemic. Methods Mixed-methods design combining three data sources: (1) quantitative data based on routine antenatal, childbirth and postnatal care data collected March 2019February 2021, (2) qualitative data from recurring rounds of semi-structured interviews conducted July 2020February 2021 with 22 maternity skilled heath personnel exploring their perceptions of service utilisation and (3) timeline data of COVID-19 epidemiology, global, national and hospital-level events. Qualitative and quantitative data were analysed separately, framed based on the timeline analysis and triangulated when reporting.

Results Three periods including a first wave, slow period and second wave were identified. Maternal health service utilisation was lower during the pandemic compared with the prepandemic year in all but one selected referral hospital. During the pandemic, service utilisation was particularly lower during the waves and higher or stable during the slow period. Fear of being infected in hospitals, lack of transportation, and even when available, high cost of transportation and service closures were key reasons affecting utilisation during the waves. However, community perception that the pandemic was over or insinuation by Government of the same appeared to stabilise use of referral hospitals for childbirth.

Conclusion Utilisation of maternal health services across the continuum of care varied through the different periods and across countries. In crisis situations such as COVID-19, restrictions and service closures need to be implemented with consideration given to alternative options for women

\section{Key questions}

What is already known?

- Prepandemic, tertiary referral hospitals were known to serve large catchment areas, many of which are urban areas, where the COVID-19 pandemic hit first in many countries.

- These facilities became even more important during the pandemic, during which pregnant women with suspected or confirmed COVID-19 could only use such facilities for care.

- For the majority of countries, the ongoing COVID-19 pandemic has progressed through different waves over time.

- Disruptions in utilisation of maternal health services across the entire continuum of care including antenatal care, intrapartum and postnatal care have been reported during the COVID-19 pandemic.

to access and use services. Information on measures put in place for safe hospital use should be communicated to women.

\section{INTRODUCTION}

The WHO declared COVID-19 a pandemic on 11 March 2020. Since then, over 240 million cases and close to 5 million deaths have been recorded globally, as of 20 October 2021. ${ }^{1}$ Over the same period, over 6 million cases and close to 150000 deaths were reported in Africa. ${ }^{2}$ After initial epidemiological data showed that there was no higher risk of contracting COVID-19 among pregnant 


\section{Key questions}

\section{What are the new findings?}

- During the pandemic, service utilisation was particularly lower during the waves and higher or stable during the slow period when the COVID-19 caseload in the community was not as high.

- Fear of being infected in hospitals, lack of transportation, and even when available, high cost of transportation and service closures were some of the main reasons that affected utilisation during the waves.

- However, community perception that the pandemic was over or insinuation by Government of the same appeared to stabilise use of referral hospitals for childbirth.

\section{What do the new findings imply?}

- When maternal health services are closed during crisis periods, such as observed during the COVID-19 pandemic, women need to be redirected and supported to access alternate sources of care.

- The reported fear of contracting COVID-19 which limited utilisation maternal health services needs to be addressed during the ongoing pandemic and in any future outbreak, with reassurance offered to women on safety of hospitals for service utilisation.

- The role and effectiveness of telemedicine in encouraging utilisation of maternal health services needs to be extensively explored, especially if health system shocks such as experienced during the ongoing COVID-19 pandemic are truly the new normal.

women than the general population, ${ }^{3}$ the global attention quickly shifted to the potential direct effect that COVID-19 posed to vulnerable populations like pregnant women. The direct effects that were of particular concern included potential increased maternal mortality, COVID-19 vertical transmission to newborns leading to stillbirths, prematurity and congenital birth defects. ${ }^{4-6}$ Early on in the pandemic, one model estimated that between 8.3 and $38.6 \%$ increase in maternal deaths per month should be expected across 118 countries. $^{7}$ Evidence gathered thus far suggests that there has been a significant increase in stillbirths and maternal deaths in low/middle-income countries (LMICs) ${ }^{8}$ Furthermore, it was estimated that there would be indirect effects which would negatively influence access, utilisation, quality and outcome of care for pregnant women. As it became apparent, these indirect effects occurred, mainly because of COVID-19 mitigation measures such as lockdowns and travel bans. ${ }^{9} 10$

Designated to primarily serve high-risk women, large tertiary referral hospitals often serve as childbirth facilities for large numbers of low-risk women seeking to avoid poor care at lower-level facilities in sub-Saharan Africa. ${ }^{711}$ Prepandemic, tertiary referral hospitals were known to serve large catchment areas, many of which are urban areas, where the COVID-19 pandemic hit first in many countries. Cities including Lagos, Nairobi and Johannesburg had been the epicentres of their respective countries. ${ }^{8}$ Also, pregnant women typically visit these referral hospitals with obstetric complications, in addition to the routine outpatient and inpatient care these facilities provide to thousands of women and newborns per annum. However, these facilities have become even more important during the pandemic, during which pregnant women with suspected or confirmed COVID-19 could only use such facilities for care. ${ }^{9}$

There have been a number of published studies that assessed effect of the COVID-19 pandemic on maternal health service utilisation in LMICs, all of which reported disruption in services during different periods of the pandemic. ${ }^{12-17}$ Among the published studies, only one has been conducted across multiple facilities and country settings, finding that there was variation in patterns of service disruptions in maternal health service utilisation in eight sub-Saharan African countries. ${ }^{13}$ Such comparative evidence is essential considering that levels of preparedness and response as well as the factors that led to disruption in maternal care service utilisation differ markedly between facilities and countries. In addition, the majority of published studies are quantitative, and gaps exist in the availability of qualitative evidence from women who needed care and skilled health personnel (SHP) who were at the frontline of the COVID-19 response. ${ }^{9}$ Combining quantitative and qualitative data will allow better understanding of factors that influenced the magnitude and duration of any disruption.

Putting all the available evidence together, we hypothesised that the impact of COVID-19 on utilisation of maternal health services will depend on the extent of the outbreak (number of reported cases and deaths) in different areas, influence of restrictive mitigation measures to the pandemic as well as the health system's preparedness (availability of trained staff, beds, intensive care units, personal protective equipment (PPE), etc). In addition, the COVID-19 pandemic has exhibited its troughs and highs with waves of cases over time. As such, it is important to adequately situate evidence within the varying contexts of how COVID-19 waves and related events may influence maternal health service utilisation across countries. The objective of this study was to prospectively assess the effect of the COVID-19 pandemic on the utilisation of maternal health services in six large referral hospitals, using a mix of deductive and inductive approaches to allow a flexible and iterative process in exploring the topic at hand. This study was nested within a larger study that explored facility-based preparedness and response to the COVID-19 pandemic in large referral hospitals in Guinea, Nigeria, Tanzania, and Uganda.

\section{METHODS \\ Study design}

Using a mixed-methods study design, this study employed three data sources which includes (1) an analysis of the timeline of key events that occurred at global, national, subnational and intrafacility levels, (2) routine hospital data before and after the WHO declared the COVID-19 pandemic and (3) semi-structured key-informant interviews (KIIs). Based on guidelines for mixed-method study design by Creswell and Clark, ${ }^{18}$ all data were collected 
Table 1 Characteristics of the participating hospitals and maternity wards before the COVID-19 pandemic

\begin{tabular}{|c|c|c|c|c|c|c|}
\hline Country & Guinea & & Nigeria & Tanzania & Uganda & \\
\hline Hospital name & $\begin{array}{l}\text { Hôpital National } \\
\text { Ignace Deen (Ignace } \\
\text { Deen National } \\
\text { Hospital) }\end{array}$ & $\begin{array}{l}\text { Hôpital Regional } \\
\text { de Mamou (Mamou } \\
\text { Regional Hospital) }\end{array}$ & $\begin{array}{l}\text { Lagos University } \\
\text { Teaching Hospital }\end{array}$ & $\begin{array}{l}\text { Muhimbili } \\
\text { National } \\
\text { Hospital }\end{array}$ & $\begin{array}{l}\text { Kawempe National } \\
\text { Referral Hospital }\end{array}$ & $\begin{array}{l}\text { Mulago Specialised } \\
\text { Women's and } \\
\text { Neonatal Hospital }\end{array}$ \\
\hline Abbreviation & HNID & HRM & LUTH & $\mathrm{MNH}$ & $\mathrm{KNRH}$ & MSWNH \\
\hline \multicolumn{7}{|l|}{ Hospital } \\
\hline $\begin{array}{l}\text { Owner/operating } \\
\text { authority }\end{array}$ & Ministry of Health & Ministry of Health & $\begin{array}{l}\text { Federal } \\
\text { Government of } \\
\text { Nigeria }\end{array}$ & $\begin{array}{l}\text { Government } \\
\text { of Tanzania }\end{array}$ & $\begin{array}{l}\text { Government of } \\
\text { Uganda }\end{array}$ & $\begin{array}{l}\text { Government of } \\
\text { Uganda }\end{array}$ \\
\hline Year of establishment & 1892 & 1908 & 1961 & 1910 & 1962 & 2019 \\
\hline Total number of beds & 467 & 90 & 761 & 1500 & 170 & 425 \\
\hline Hospital designation & $\begin{array}{l}\text { Tertiary teaching } \\
\text { hospital }\end{array}$ & Regional hospital & $\begin{array}{l}\text { Tertiary teaching } \\
\text { hospital }\end{array}$ & $\begin{array}{l}\text { Tertiary } \\
\text { teaching } \\
\text { hospital }\end{array}$ & $\begin{array}{l}\text { Maternity/women's } \\
\text { care and neonatal } \\
\text { care }\end{array}$ & $\begin{array}{l}\text { Maternity/women's } \\
\text { care and neonatal } \\
\text { care }\end{array}$ \\
\hline $\begin{array}{l}\text { Private services } \\
\text { offered }\end{array}$ & No & No & Yes & Yes & Yes & Yes \\
\hline \multicolumn{7}{|l|}{$\begin{array}{l}\text { Maternity services and } \\
\text { infrastructure }\end{array}$} \\
\hline $\begin{array}{l}\text { Routine outpatient } \\
\text { antenatal care }\end{array}$ & $\begin{array}{l}\text { No (only for } \\
\text { complicated cases) }\end{array}$ & Yes & Yes & Yes & Yes & Yes \\
\hline Childbirth & Yes & Yes & Yes & Yes & Yes & Yes \\
\hline $\begin{array}{l}\text { Routine outpatient } \\
\text { postnatal care }\end{array}$ & No & No & Yes & Yes & Yes & Yes \\
\hline Caesarean sections & Yes & Yes & Yes & Yes & Yes & Yes \\
\hline $\begin{array}{l}\text { Private maternity } \\
\text { services offered }\end{array}$ & No & No & Yes & Yes & Yes & Yes \\
\hline \multicolumn{7}{|l|}{$\begin{array}{l}\text { Service outputs (pre- } \\
\text { COVID-19) }\end{array}$} \\
\hline $\begin{array}{l}\text { Number of outpatient } \\
\text { antenatal visits (annual } \\
\text { for 2019) }\end{array}$ & - & 1148 & 5860 & 23048 & 35169 & 5216 \\
\hline $\begin{array}{l}\text { Number of births } \\
\text { (annual for 2019) }\end{array}$ & 5927 & 3468 & 933 & 7385 & 21778 & 1163 \\
\hline
\end{tabular}

in parallel, analysed separately and triangulation of the findings at the synthesis stage allowed for an in-depth understanding of the situation in the six hospitals.

\section{Hospital selection and profiles}

The six hospitals were purposively selected, with emphasis placed on hospitals with large referral maternity wards in urban areas of different sub-Saharan African countries (two in East Africa, two in West Africa, including a francophone country) were selected. The participating hospitals were Hôpital National Ignace Deen/ Ignace Deen National Hospital (HNID) and Hôpital Regional de Mamou/Mamou Regional Hospital (HRM) in Guinea, Lagos University Teaching Hospital (LUTH) in Nigeria, Muhimbili National Hospital (MNH) in Tanzania, Kawempe National Referral Hospital (KNRH) and Mulago Specialised Women's and Neonatal Hospital (MSWNH) in Uganda; their profiles based on information collected from each hospital's primary investigator (PI) are shown in table 1.

\section{Data and analysis}

\section{Key events}

Initial data regarding key events were collected during the semi-structured KIIs conducted as part of this study. Insights from the interviews helped with establishing the time range of interest (1 January 2020 to 28 February 2021). Subsequently, a pro-forma tool was designed to capture events that could influence service provision and utilisation at the participating hospitals, and on national and global levels. At hospital-level, data collected included any periods of maternity services closure and significant modifications to service delivery that could alter utilisation. These data were completed by the country PIs and hospital co-PIs. National level events included periods of national lockdown(s), curfews and travel ban. These also included other key events that were aimed at or have an established potential to alter behaviour of maternity service users such as introducing subsidies for user fees, tax credits or cash schemes. These data were sourced from the Oxford COVID-19 
Government Response Tracker, Blavatnik School of Government and University of Oxford..$^{19}$ One-off events were distinguished from extended ones. National timelines were verified for accuracy and expanded with contextual detail by the country PIs and hospital co-PIs and through review of weblinks describing the national events. Numbers of cases and deaths due to COVID-19 at the national level were collected from 'Our World in Data' (https://ourworldindata.org/covid-deaths) and validated on the WHO COVID-19 dashboard (https:// COVID-19.who.int/). Global events were sourced from the WHO's COVID-19 response timeline (https://www. who.int/emergencies/diseases/novel-coronavirus-2019/ interactive-timeline). Using epidemiological week cutoffs defined by Salyer $e t a l,{ }^{20}$ and information gathered by country PIs and hospital co-PIs, we divided the timeline for each country into three phases: first wave, slow period and second wave. This was based on weekly incidence of COVID-19 cases in the study countries. For Tanzania, which did not report epidemiological data after June 2020 , we used periods observed in countries in proximity to it. National data on COVID-19 cases and deaths were entered into Microsoft Excel and presented as line charts; all events per country were mapped on a timeline visual.

\section{Routine data}

Monthly aggregated routine health statistics (from 1 January 2019 to 28 February 2021) were collected from each of the participating hospitals by clinical researchers in collaboration with hospital-based clinicians and data clerks between 1 June 2020 and 28 March 2021. We analysed three routine indicators which represent main aspects of maternal care utilisation: number of outpatient antenatal care (ANC) visits, number of births and number of outpatient postnatal care (PNC) visits. A detailed list of routine statistics indicators and their definitions is included in online supplemental material 1. The aggregate routine data used for calculation of these indicators were extracted from multiple sources within each hospital (eg, labour ward registers, medical records, health management information system, etc) and the number of sources ranged between two and four per hospital (online supplemental material 1). When multiple data sources for the same indicator were available, data were collected from all sources and validated against each other. In case of discrepancy, the researchers included the numbers from the most reliable source according to the hospital PIs and data clerks.

Aggregated monthly data were inputted in a standardised Microsoft Excel sheet. In collaboration with the researchers from each country, data review and verification were conducted a minimum of two times for each hospital. This was done to allow for detection of missing values and outliers in each of the selected hospitals. We conducted descriptive analysis of each indicator for a period of 24 months, divided into two 12-month time periods representing a year before the pandemic was declared (from March 2019 to February 2020 labelled as
pre-COVID-19) and a year afterwards (from March 2020 to February 2021 labelled as during COVID-19). Frequencies were displayed in line charts. Indicator values for the two time periods were compared, matched with other key events and triangulated against findings from semistructured interviews.

\section{Interviews}

We conducted repeated semi-structured interviews with SHPs who were only included in the study if they principally worked in maternity. We adopted a purposive sampling of key informants to ensure maximum variation in the experiences of SHP of varying seniority levels (junior and senior staff) and cadres (medical doctors, midwives and nurses). Potential participants were first approached by the hospital PIs; if they agreed to be interviewed, they were compensated for their time and use of mobile data. Data were collected over one to four rounds of interviews conducted between July 2020 and February 2021. We interviewed two to six maternity SHP in each participating hospital per round. At LUTH (Nigeria) and MNH (Tanzania), we interviewed four respondents each. At KNRH (Uganda) and MSWNH (Uganda) we interviewed two people each. In HNID (Guinea) we interviewed six people and in HRM (Guinea), we interviewed four key informants. In total 22 SHPs were interviewed, and 50 interviews took place.

We used a semi-structured interview guide to comprehensively capture information related to changes in the processes of care utilisation across all hospitals and time-points. The content of this guide was developed to record and understand perceptions of respondents on shifts in maternity case volumes, as well as any observations on influence of COVID-19 measures on service utilisation. Interviews lasting between 20 and $120 \mathrm{~min}$ were conducted by two researchers virtually using Zoom (Zoom Video Communications, San Jose, California, USA) for Nigeria, Tanzania and Uganda (LB), and faceto-face in Guinea (ND). All interviews were recorded and transcribed in the language of the interview (English or French), de-identified, and imported into the computerassisted qualitative data analysis software Dedoose. Analysis was an iterative process which was done concurrently with data collection. This approach allowed the researchers to adapt the interview guide in the repeated interview rounds based on respondents' answers to the previous rounds and to the country situation.

Data analysis was conducted using the framework method. ${ }^{21}$ Following familiarisation with the data by re-listening to the recordings and reading the transcripts which was done by three researchers (ND, AS and LB), coding of the first six interviews was independently done, from which emerging codes were identified (inductive) keeping the structure of the interview guide in mind (deductive). A coding tree was subsequently developed and systematically applied to the interviews by one researcher and checked by another. The themes and examples emerging from the interviews were mapped on 
to a matrix by period (first wave, slow period and second wave) ${ }^{20}$ Emerging themes were further summarised to capture similarities and differences across the three periods and six hospitals, and to identify relationships between the main themes in the data.

\section{Triangulation and synthesis}

The triangulation and synthesis of data from the three data sources were conducted in an iterative, prospective and collaborative manner, first by sharing and discussing findings during 21 biweekly research team meetings between May 2020 and March 2021, and on completion of all data collection in April-July 2021. Time trends observed in the routine data indicators were compared against findings from the key event analysis and the qualitative data, including perceptions of SHPs on service utilisation, to identify and discuss intersections between all three data sources. We present the findings for each period using all three data sources.

\section{Patient and public involvement}

Patients and/or the public were not involved in the design, conduct, reporting, or dissemination of this research.

\section{RESULTS}

As per all the available evidence, the first wave in Guinea started 23 March 2020 and ended 3 August 2020. Since then, the country transitioned to a slow period and remained in this period till the end of our study. For Nigeria, the first wave was from 16 March to 28 June 2020, followed by a slow period till 16 November 2020 and a second wave from this date till the end of our study in February 2021. For Uganda, the first wave started 4 May 2020, though restrictive measures were already in place from 1 March 2020. The first wave continued till 7 June, followed by a slow period till 3 August 2020 after which a second wave commenced till January 2021. For Tanzania, the waves were estimated based on a combination of reports from respondents, extrapolation from events within the country and the time periods used in neighbouring countries. The first wave extended from 16 March to 28 June 2020, and the slow period followed from July to November 2020. In December 2020, country team and respondents reported a perceived increased in the spread of the virus nationally, indicating the beginning of the second (undeclared) wave, when no national restriction measures were witnessed.

\section{First wave}

Timeline of key events related to utilisation along with COVID-19 cases and deaths are shown in figure 1 . In Guinea, the government instituted travel restrictions by 21 March 2020 and declared a state of emergency and a domestic travel ban including a ban on travel between Conakry and other cities in the deep country on 26 March 2020. During this period, there were 59 cases on average per week. In Nigeria, bans on international travel and mass gatherings were instituted by 18 March 2020 and a national lockdown including a domestic travel ban was put in place by 30 March 2020. New daily COVID-19 cases in the country continued to rise until mid-June. Despite this, the nationwide lockdown was lifted 1 June 2020. Domestic travel ban was subsequently lifted 8 July 2020. During this wave, there were an average of 314 COVID-19 cases per week. At LUTH (Nigeria), obstetric emergency services were initially closed between 20 and 29 April while maternity were shut down between 6 May and 1 June. In Tanzania, the Government declared that there will be no lockdown, though there was a ban on mass gathering between 20 March and 1 June 2020 and travel restrictions between 11 April and 18 May 2020. During this wave, there was an average of 11 cases officially notified every week. In Uganda, a domestic travel ban was instituted on 23 March 2020 and another on mass gathering by 1 April 2020. Despite this, election campaigns continued in the country. From 26 March to 30 May 2020, outpatient clinics were closed at MSWNH (Uganda).

From the routine data, during the first wave there was a decrease in ANC outpatient consultations in April and May 2020 compared with the same period of the previous year in LUTH (Nigeria), MNH (Tanzania), KNRH and MSWNH (Uganda). In HRM (Guinea), a decline was observed in May 2020 compared with the previous year (figure 2). The number of deliveries in HNID (Guinea) and KNRH (Uganda), was similar during the first wave compared with pre-COVID-19. In HRM (Guinea) and MSWNH (Uganda), the number of deliveries was higher during the COVID-19 pandemic compared with the previous year. In $\mathrm{MNH}$ (Tanzania) and LUTH (Nigeria) there was a decrease in the number of deliveries during the first wave compared with the previous year, with a sharp decrease observed in LUTH (Nigeria) to 16 and 27 deliveries in May and June, respectively (figure 3). Attendance to $\mathrm{PNC}$ consultations was lower during the first wave compared with pre-COVID-19 in all hospitals, except for an increase in June 2020 in MNH (Tanzania). Data were not available in April and May 2020 from LUTH (Nigeria) (figure 4). Based on qualitative data from the interviews, during first wave of COVID-19, respondents' perception of utilisation of maternal care services was that there were fewer women using outpatient (ANC and PNC) and inpatient (childbirth) services. According to respondents, the decline in use of care was happening in contexts of high pre-existing levels of poverty exacerbated by reductions of income and more expensive transport during lockdowns, making care-seeking unaffordable to many women: 'what I think, it is not COVID-19 that is preventing them [women] from coming in [to the hospital]. It is transport fares. Transport fares were increased [...]' (Respondent C, MSWNH, Uganda). Women and their families were also thought to be actively avoiding hospitals due to fear of infection, and this was thought to particularly affect maternity wards in hospitals which had COVID-19 treatment centres onsite, such as LUTH. For example, one respondent from MNH said, 'I know most of the women were 


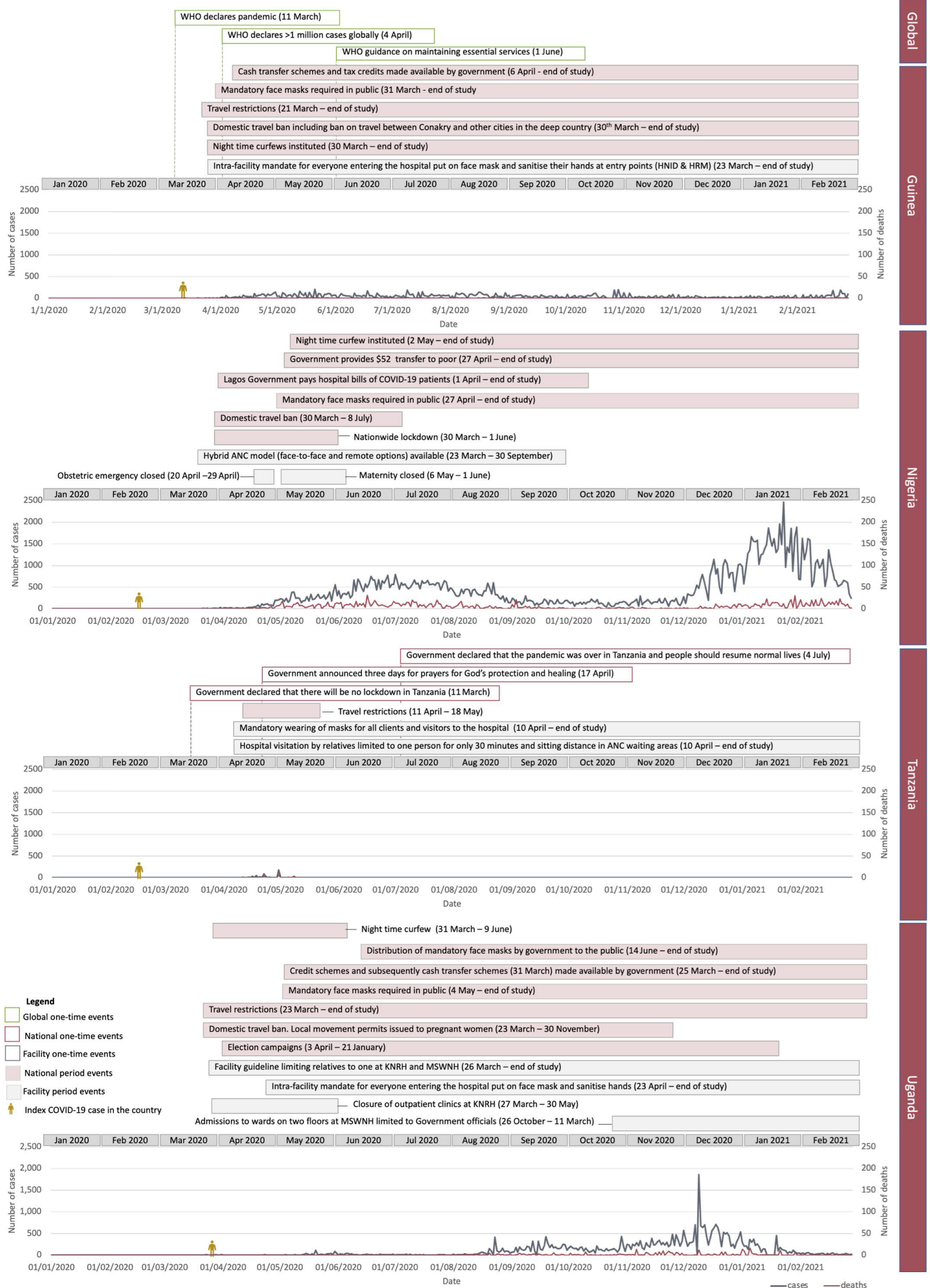

Figure 1 Timeline of key events at global, national and facility levels. ANC, antenatal care; HNID, Hôpital National Ignace Deen/Ignace Deen National Hospital; HRM, Hôpital Regional de Mamou/Mamou Regional Hospital; KNRH, Kawempe National Referral Hospital; MSWNH, Mulago Specialised Women's and Neonatal Hospital. 


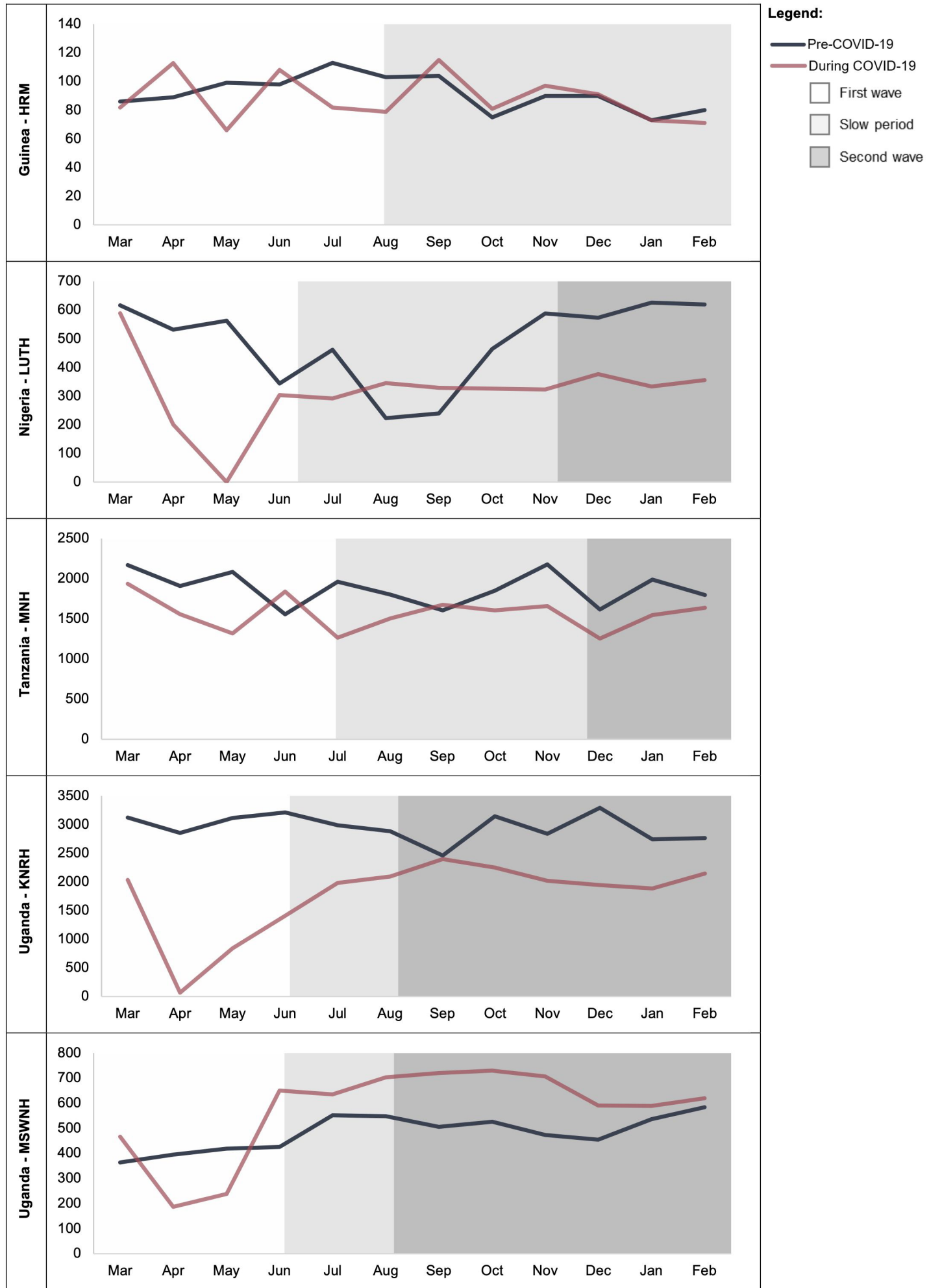

Figure 2 Number of outpatient antenatal care consultations by month in each referral hospital before and during the COVID-19 pandemic. Outpatient service not offered by Hôpital National Ignace Deen in Guinea. HRM, Hôpital Regional de Mamou/Mamou Regional Hospital; KNRH, Kawempe National Referral Hospital; LUTH, Lagos University Teaching Hospital; MNH, Muhimbili National Hospital; MSWNH, Mulago Specialised Women's and Neonatal Hospital.

afraid of big hospitals. Like I even had a patient of mine who has delivered four children at Muhimbili and then she was so scared of COVID-19 that she asked me if I attend any other private clinic and I said yes, she came ...' (Respondent C, MNH, Tanzania). Also, women were deemed not to have been using LUTH (Nigeria) because the maternity ward was temporarily closed due to high number of staff self-isolating. In Uganda, SHP reported that many women could not get to hospitals because of the strict lockdown and travel ban, while some, especially those seeking ANC, 


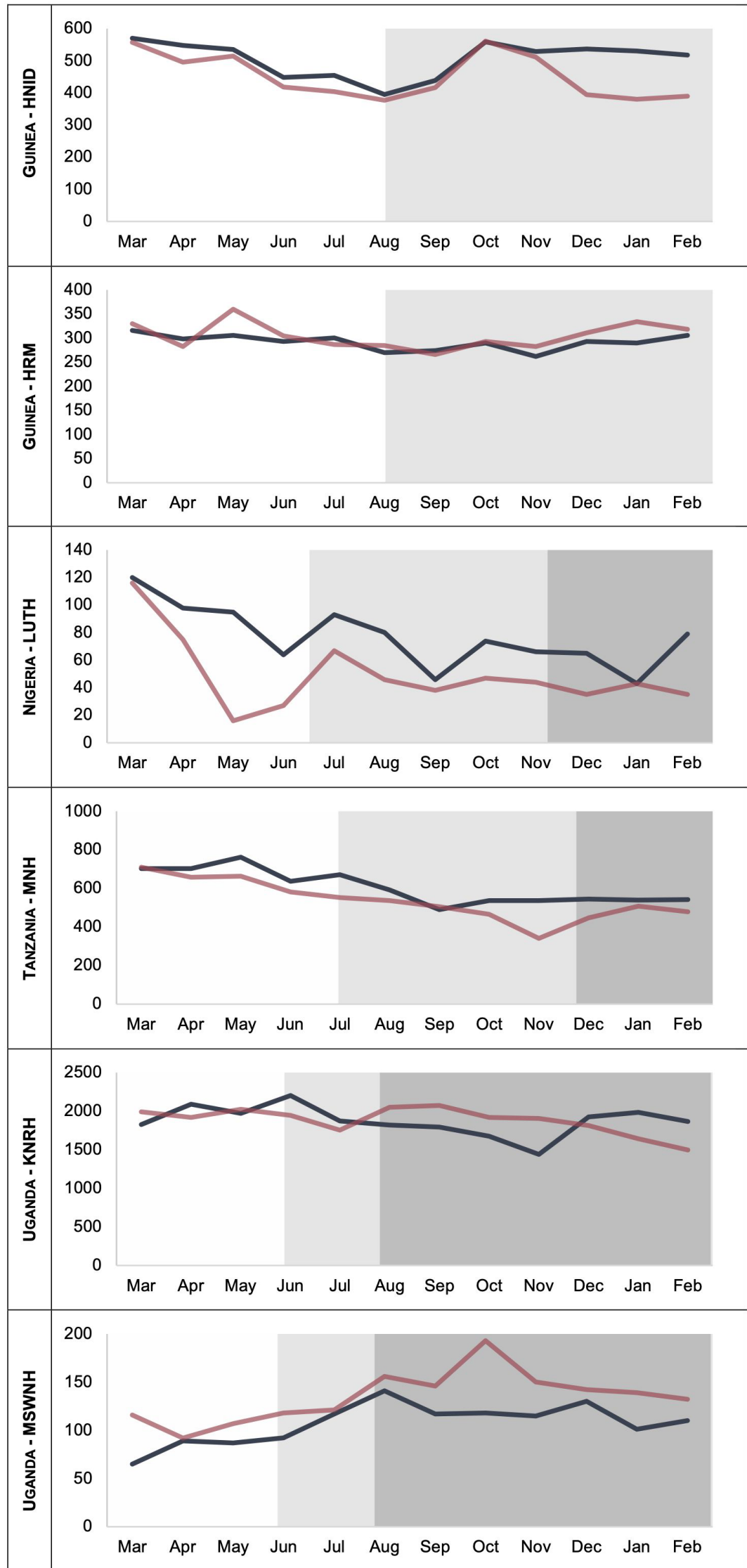

Legend:

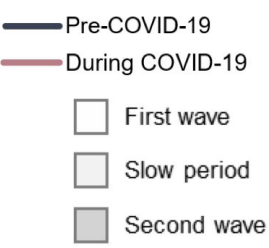

Figure 3 Number of deliveries by month in each referral hospital before and during the COVID-19 pandemic. HNID, Hôpital National Ignace Deen/Ignace Deen National Hospital; HRM, Hôpital Regional de Mamou/Mamou Regional Hospital; KNRH, Kawempe National Referral Hospital; LUTH, Lagos University Teaching Hospital; MNH, Muhimbili National Hospital; MSWNH, Mulago Specialised Women's and Neonatal Hospital. 


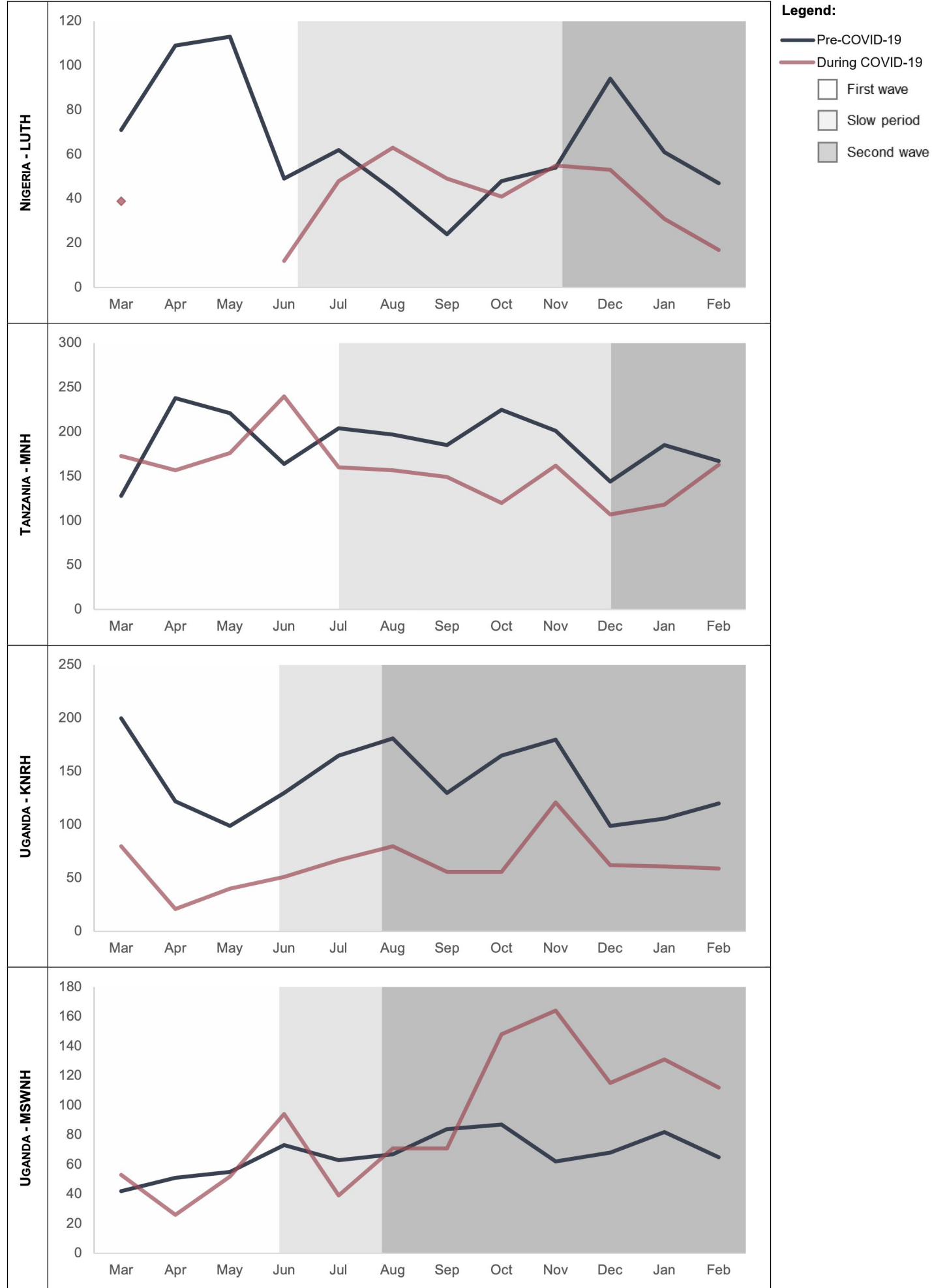

Figure 4 Monthly number of postnatal care consultations for women in each referral hospital before and during the COVID-19 pandemic. KNRH, Kawempe National Referral Hospital; LUTH, Lagos University Teaching Hospital; MNH, Muhimbili National Hospital; MSWNH, Mulago Specialised Women's and Neonatal Hospital.

did not bother going to hospitals because they were unsure if they were open.

\section{Slow period}

From the timeline, in Guinea, there were 36 cases per week on the average. Travel restrictions, night-time curfews and ban on mass gatherings continued from the first wave all through this period. In Nigeria, there were 211 cases per week averagely. No nationwide lockdown or domestic travel ban was instituted during this period. In Tanzania, as of 4 July 2020, the Government declared 
that the pandemic was over, and people could resume their normal lives. No cases of COVID-19 were reported during this phase. In Uganda, there were nine cases per week averagely during this period. Travel restrictions remained though local movement permits were issued to pregnant women.

As per the routine data, the decrease in ANC outpatient consultations continued from the first period into July, and August 2020 in HRM (Guinea), compared with the previous year (figure 2). In LUTH (Nigeria), the number of ANC consultations stabilised during this period while at KNRH (Uganda), the number of ANC consultations increased during this period but did not reach the same level as prepandemic in both facilities. In MSWNH (Uganda), the number of ANC consultations increased to a higher level than before the pandemic starting in June 2020. In MNH (Tanzania), overall, the number of ANC consultations was lower during the pandemic compared with before, apart from June 2020. The number of deliveries in the health facility was stable during this period in HRM (Guinea). The number of deliveries was lower during the COVID-19 pandemic compared with the previous year in HNID (Guinea), LUTH (Nigeria) and MNH (Tanzania). In KNRH (Uganda), the number of deliveries per month increased at the end of the slow period in August 2020. As with the first period, MSWNH was the only hospital where the number of births was higher during these pandemic months compared with same months before (figure 3). For PNC during the slow period, the number of consultations was lower in $\mathrm{MNH}$ and KNRH compared with pre-COVID-19. In LUTH, the number of PNC consultations was higher in September 2020 compared with the previous year but dipped to lower than the prepandemic numbers in October 2020. The trends varied month-to-month in MSWNH during the slow period (figure 4).

During the slow period, respondents noted that the number of users appeared to have recovered slightly, although not to pre-COVID-19 levels. In Tanzania and Uganda, this period coincided with a perceived annual 'low season' of births where lower numbers of maternity patients are seen. In Uganda, publicity around lockdown's impact on women seeking care helped in making a case to the national government to exempt pregnant women from the travel ban. This was supported with evidence suggesting that women were having more complications by not being able to access hospitals, with one respondent describing the role SHPs played in this as,

our involvement was the mothers who show these complications, so, who had come, but they had already developed complications; they have lost babies, or they had a complication as fistula or sepsis. They are staying at the hospital for a long period. So, we had documented those. We shared those with the ministry of health. They used part of that to justify when they report to the minister. The minister I think took it to the cabinet to justify why pregnant mothers should be allowed to come, to go to the hospital, to go for antenatal without needing to have a permission from anywhere (Respondent D, MSWNH, Uganda).

According to SHPs, unaffordability of transport remained an issue for some women during the slow period because lockdown exacerbated poverty in Nigeria, consequently exacerbating women's inability to seek care. However, also in Nigeria, some pregnant women were noted to have transferred to LUTH after having started ANC in different/private facilities during first wave. One SHP at LUTH (Nigeria) said

You know the challenge before is because a lot of people were afraid of coming to LUTH because we have a COVID centre. So many of the women, those that were not coming, actually they were going to private hospitals. Some of them are now coming to register in LUTH, but they started their antenatal in private hospitals and then now coming here because they feel the threat is less (Respondent C, LUTH, Nigeria).

In Guinea, it was a general perception that the number of women attending outpatient and inpatient services had not been affected much and utilisation had returned to normal (HNID and HRM, Guinea).

\section{Second wave}

In Nigeria, there was a larger second COVID-19 wave than the first, starting from early December 2020 till February 2021. Average number of weekly COVID-19 cases was 894. Schools were closed during this period but there was no restriction of movement. In Uganda, the domestic travel ban remained till mid-November, pregnant women continued to use local permits while travelling to a hospital. Average number of weekly COVID-19 cases was 211. In Tanzania, no cases of COVID-19 were reported. There had no second wave in Guinea at the end of this study.

During the second wave, the number of ANC consultations was lower than the same period in the preCOVID-19 year in LUTH (Nigeria), MNH (Tanzania) and KNRH (Uganda). In MSWNH (Uganda), the number of ANC consultations declined during the second wave in comparison to the slow period but remained higher than the pre-COVID-19 levels. For childbirth, the number of deliveries was lower during the COVID-19 pandemic compared with the previous year in LUTH (Nigeria), MNH (Tanzania) and KNRH (Uganda). On the other hand, the number of deliveries was higher in MSWNH (Uganda) during the second wave compared with the same period in the pre-COVID-19 year, with a noted increase to 193 births in October 2020. For PNC, the number of consultations was lower compared with preCOVID-19 months in LUTH (Nigeria), MNH (Tanzania) and KNRH (Uganda). Contrarily, in MSWNH (Uganda), the number of PNC consultations was higher during the pandemic compared with the previous year.

In the second wave, there was a notable variability in the perception of care utilisation in the participating hospitals, which coincided with a high season of births in 
Uganda and Tanzania. In Uganda, according to SHPs, this period coincided with a 'high season' or 'boom months', which usually saw a rise in numbers of births. SHPs had a perception that there was a large number of women who conceived during the first lockdown who were now using the hospitals to give birth. However, SHPs opined that the volume of pregnant women seeking care had not completely recovered to prepandemic levels as transport cost was much more expensive compared with before COVID-19. In Tanzania, more women appeared to be attending routine ANC with one SHP saying, 'For those who come to antenatal clinic, few would ask you, like "I don't feel anything new, is it safe to come to the hospital now with this COVID-19?" very few, but the majority are just coming as usual' (Respondent C, MNH, Tanzania). In Nigeria, respondents reported that women were once again afraid to seek care in health facilities but women who needed care came to hospitals and were less likely to opt for private sector than in first wave. In Nigeria also, SHPs reported that they observed a lower uptake of outpatient PNC as women avoided if no issues, and SHPs as well as women preferred telemedicine appointments in such situation.

\section{DISCUSSION}

This paper explores maternal health service utilisation before and during the COVID-19 pandemic in six referral hospitals in sub-Saharan Africa, using prospectively collected data from three sources. Our findings show that utilisation of maternal health services across the continuum of care varied through the different periods and across countries. However, some similarities exist in emergent patterns and factors that influenced utilisation. First, except for MSWNH (Uganda), maternal health service utilisation in all selected hospitals was generally lower during the pandemic than the prepandemic year. During the pandemic itself, service utilisation was particularly lower during the waves and higher or stable during the slow period. Fear of being infected in hospitals, lack of transportation, and even when available, high cost of transportation and service closures were some of the main reasons that affected utilisation during the waves. However, perception that the pandemic was over by the community or insinuation by Government of the same appeared to stabilise use of referral hospitals for childbirth.

During the first wave, like all but one of the countries in our study, many countries in sub-Saharan Africa instituted very restrictive measures to try to slow down the spread of COVID-19. ${ }^{22}$ In all countries where lockdowns were instituted, there were economic disruptions and untold hardship reported by families. In Tanzania, where there was no lockdown, students and young children were mandated to stay at home. ${ }^{22}$ This also meant that mothers, many of whom are the primary caregivers, had to stay at home with the children. Across all countries, the lockdown and travel ban essentially also meant that means of transport were not available for women seeking maternal care. Even when available, some SHPs reported that the cost of transportation was high. A previous study reported a high cost of service utilisation during the first wave of COVID-19 in Nigeria, not just as a result of transportation, but also in the actual cost of care including purchasing PPE that they needed to purchase to support their care. ${ }^{23}$ Even before the pandemic, many women already faced significant financial burden in using maternal health services in LMICs. ${ }^{24}$ As such, any additional costs in a crisis period will only further exacerbate inequalities. In addition, as reported by SHPs in our study, within the communities, there was fear among pregnant women of contracting COVID-19 if they used a health facility. This view has been expressed by other SHPs and women themselves in many LMICs. ${ }^{9} 1015$ As evident in our study, there was a decline in women attending ANC and deliveries at HRM (Guinea), LUTH (Nigeria), MNH (Tanzania), KNRH (Uganda) and MSWNH (Uganda). This period coincides with the first period of significant rise in COVID-19 cases in the countries. Similar decline in service utilisation was observed in other LMICs including India and Rwanda. ${ }^{15} 16{ }^{25}$ However, as will be expected, any facility closures, like that of LUTH (Nigeria) from 6 May to 1 June brought the lowest number of service use for ANC and delivery. Closure of outpatient clinics at and KNRH (Uganda) from 27 March to 20 May meant no ANC utilisation during this period. However, when facilities remained open and functional, many women gave birth in those hospitals though the numbers were generally less than the pre-pandemic period.

In the slow period, for the most part, number of ANC visits and births stabilised though it did not quite reach prepandemic numbers in many hospitals. It aligned with a period in which average daily COVID-19 cases were lower compared with the first period, fear was generally reduced, travel restrictions and ban on local movement removed or exception given to pregnant women and a perception that the pandemic was over in some countries. This is not a period that is widely described in the wider literature. ${ }^{10}$ However, it is important to highlight here that a perception of the pandemic being over whether self-perceived or government-induced appears to have been sufficient for change in utilisation patterns. To be clear, COVID-19 cases were down, but the pandemic was not over. In sub-Saharan Africa, including three of our study countries (Guinea, Tanzania and Uganda), the politicisation of the public health response altered the perception of the population. ${ }^{26}$

By the second wave, despite rising cases in many African countries, several countries had limited stringent public measures in place. ${ }^{20}$ As per our results, service utilisation across the continuum of care was lower in many public hospitals compared with the slow period and the prepandemic year. Without lockdowns and travel bans in this period, fear and high transportation cost were still reasons given by SHPs for women not using services. During this period, SHPs did highlight that some women 
came to the hospital needing reassurance that they were safe from COVID-19.

Across both first and second waves, the common pattern is reduction in service utilisation-the SHPs perceived this, and the routine data supports them. Through the entire period, the perceptions about case load (ie, utilisation of ANC and delivery care) are aligned with the trends observed in the routine data-which are mostly a decrease in attendance during the first months of the pandemic, followed by a return to prepandemic or increase. One exception was MSWNH (Uganda) because in this hospital the comparison of trends with the previous year is not very accurate since the hospital opened in 2019. One other finding common to both waves was fear of contracting COVID-19 in hospitals. In our study, utilisation of maternal health services varied depending on COVID-19 developments in country (epidemiological and political), role of hospital in country response and role of maternity ward in caring for women-inclusive of all women, and COVID-19 women in particular. Uganda ranked first in Africa in terms of suppressing the COVID-19 pandemic in August 2020, according to the Lancet COVID-19 Commission. ${ }^{27}$ While there were other reasons explaining the pattern of service utilisation in Uganda, utilisation numbers were mostly stable or improved after an initial fallout from the lockdown. In Tanzania, ANC visits started rising again in July after the Government declared that the pandemic was over, and people should resume their normal lives. In terms of roles of hospital during the pandemic, some were formal (HNID (Guinea) officially designated COVID-19 maternity) or informal (LUTH (Nigeria) had experience with first COVID-19 case in country-became an informal hub for training, sharing, care provision). In both cases, SHPs associated this label as a reason for fear among women. On the other hand, hospitals like MNH (Tanzania) that only made procedural changes like mandating face masks, spacing of ANC visits, etc, without a COVID-19 label attached to them, also appeared to mostly report stable numbers of service utilisation. In addition, HRM (Guinea) was hardly affected by COVID-19, as seen in the routine data. In addition to not having a label, as the hospital never even handled a COVID-19 positive woman, this might have also been due to the fact that the country did not go through a second wave during the study period. Evidence shows that amid the pandemic, pregnant women's worry, among many other factors that were already cause for worry prepandemic, were mostly related to their fear of COVID-19 itself and having a relative infected with COVID-19. ${ }^{28}$

There are some strengths of our study. First, it is a uniquely collaborative work capturing complexity of how maternity wards in large referral hospital experienced to the challenges of COVID-19 in the first year of the pandemic. In collecting data prospectively, we were also able to collect key events on hospital/maternity ward levels, as these do not seem to be reported or collated anywhere, unlike national events for which a number of databases were set up immediately. Second, our study involved triangulation of three types of data across two waves and a slow period of the pandemic. The richness in the different types of data and country settings allowed us to make some generalisations and draw pertinent lessons. On the other hand, there are limitations to consider in interpreting our findings. First, we did not include women in the research mostly due to travel restrictions in the middle of the COVID-19 crisis and budget constraints. However, SHPs who were on the frontline throughout the crisis and experiencing first-hand the challenges of their patients using care were able to capture some of the factors linked to changes in maternal service utilisation in hospitals. ${ }^{9}$ In addition, we were unable to fully explore the role of subsidies and conditional cash transfer schemes like those implemented in Guinea and Nigeria, in promoting maternal health service utilisation, as the SHPs could not detail how it had helped. Third, we were not able to travel to the field ourselves to conduct the interviews. However, online platforms, which also became widely used during the initial phases of the pandemic were used for the interviews complemented by extensive team engagement of research team members working in-country. Fourth, we were unable to accurately delineate the period of waves for Tanzania, as data reporting from the country was stopped in May 2020.

This research has clear implications for policy especially as it relates to preparedness for future health system shocks. Efforts need to be made to keep maternity units safely open for as long as is feasible, as was done in Canada during the severe acute respiratory syndrome outbreak in $2003 .{ }^{29}$ When services in referral hospitals have to close, women need to be actively redirected to safe alternatives of care. Telemedicine may offer a nonphysical contact alternative to care. Indeed, the role of telemedicine in encouraging utilisation of maternal health services needs to be extensively explored, especially if health system shocks like COVID-19 are truly the new normal. This should be done while recognising the double-edged nature of telemedicine as regards its merits and demerits. ${ }^{29}$ There is also a need for clarity regarding if telemedicine appointments count as service used or a stop gap in the middle of a crisis. In LUTH (Nigeria), which was the only facility in our study to implement telemedicine, telemedicine consultations were not counted as part of ANC outpatient consultations.

However, in these situations, politics of being closed influences decisions on appropriate actions. Maternity wards are usually profitable to public hospitals, and closures or lower volumes can be financially (and politically) harmful, especially at a time of higher expenditures on PPE etc. Fear of contracting COVID-19 was noted as a reason for pregnant women not seeking care. Yet, large urban hospitals like those included in our study were the only sources of care for thousands of women during the COVID-19 pandemic in parts of sub-Saharan Africa. However, this fear experienced by women reported in this study, and experienced by SHPs themselves, ${ }^{30}$ is not 
new. It was the same fear that kept women away from the hospitals during the Ebola Virus Disease outbreak in West Africa. ${ }^{31}$ What is different this time around with COVID-19 is that the fear of SHPs on treating women was not as high as with Ebola Virus Disease. It might be because of the comparative case fatality, accumulated knowledge and experience from dealing with previous outbreaks, or simply their heroism faced with a crisis. As such, women who used referral hospitals were likely to receive care from SHPs who were not as fearful. This probably explains why overall high satisfaction with maternal health service received by women who used services during the outbreak. ${ }^{32}$ In any case, fear within the community towards using referral hospitals, which were the cornerstone of the COVID-19 response in many LMICs must be addressed. This can be backed up with supporting promotion campaigns using narratives of satisfaction with care of women who used services during the crisis.

\section{CONCLUSION}

Our study across six hospitals in four sub-Saharan African countries shows that the pandemic affected utilisation of maternal health services in 'waves and troughs'. In the first wave, there was an initial dip in service utilisation due to fear and restrictions, a trough during which utilisation recovered, though not to the same extent compared with pre-COVID-19 and a second wave where it dipped again, though not to the depths seen in the first wave. The in-depth understanding of these six hospitals collected in this study shows that the decline in utilisation were related to lockdown restrictions, health service closures and initial fear of infections in hospitals (first wave) rather than the actual number of COVID-19 cases in a country (second wave). It is clear that the perceived risk of contracting the disease in hospitals and restrictive measures without consideration for pregnant women were the key drivers that influenced service utilisation. Though the world is attempting to now live with COVID19 , it is important to continue to monitor service utilisation through the different phases of the pandemic, including impact of vaccination on service utilisation during the pandemic. Going forward, in crisis situations such as COVID-19, restrictions such as lockdowns, curfews, etc, and service closures need to be implemented with consideration given to alternative options for women to access and use services while providing clear reassurance of measures put in place to make hospitals safe for use.

\footnotetext{
Author affiliations

${ }^{1}$ School of Human Sciences, University of Greenwich, London, UK

${ }^{2}$ LSE Health, London School of Economics and Political Science, London, UK ${ }^{3}$ Department of Public Health, Institute of Tropical Medicine, Antwerpen, Belgium ${ }^{4}$ Department of Obstetrics and Gynaecology, School of Medicine, College of Health Sciences, Makerere University, Kampala, Uganda

${ }^{5}$ Department of Health Policy Planning and Management, Makerere University School of Public Health, Kampala, Uganda

${ }^{6}$ Department of Obstetrics and Gynaecology, College of Medicine, University of Lagos, Idi-Araba, Lagos, Nigeria
}

${ }^{7}$ Centre National de Formation et de Recherche en Santé Rurale de Maférinyah, Forécariah, Guinea

${ }^{8}$ Muhimbili University of Health and Allied Sciences, Dar es Salaam, Tanzania, United Republic of

${ }^{9}$ Kawempe National Referral Hospital, Kampala, Uganda

${ }^{10}$ Mulago Specialized Women and Neonatal Hospital, Kampala, Uganda

${ }^{11}$ Maternité de I'Hôpital National Ignace Deen de Conakry, Conakry, Guinea

${ }^{12}$ Maternité de l'Hôpital Régional de Mamou, Mamou, Guinea

${ }^{13}$ Department of Obstetrics and Gynaecology, Muhimbili National Hospital, Dar es Salaam, Dar es Salaam, Tanzania, United Republic of

${ }^{14}$ Centre d'Excellence Africain pour la Prévention et le Contrôle des Maladies Transmissibles, Université Gamal Abdel Nasser de Conakry, Conakry, Guinea

Twitter Aduragbemi Banke-Thomas @abankethomas, Aline Semaan @semaaline, Bosede Bukola Afolabi @Coolgynae and Lenka Benova @lenkabenova

Acknowledgements We would like to thank maternal and newborn healthcare providers who contributed their valuable time to participate in the interviews on repeated occasions despite ongoing difficult circumstances and high workload. We acknowledge Ms Kristien Wynants and Ms Nadine El Rashidi for their support in interview transcription. We also acknowledge all the Institutional Review Committees for providing helpful suggestions on this study protocol, and for the expedited review of this study. We are particularly indebted to all the health workers working at the frontline who took part in this research. They are the true heroes of the pandemic.

Contributors Conceptualisation, investigation, data curation, formal analysis, visualisation, writing —original draft preparation, writing — review and editing: AB-T. Conceptualisation, data curation, formal analysis, visualisation writing —original draft preparation, writing — review and editing: AS. Investigation, data curation, validation, writing — review and editing: DA, OB, ND, AK, SN, 00, MA, WAA, ADiallo, LD, MCD, $\mathrm{CM}$, NM, TS. Conceptualisation, funding acquisition, project administration, writingreview and editing: TD, ADelamou. Conceptualisation, project administration, validation, writing — review and editing: BBA, AN, AP. Conceptualisation, funding acquisition, project administration, methodology, investigation, data curation, formal analysis, writing — original draft preparation, writing — review and editing, and takes full responsibilty for the overall content, conduct of the study, had access to the data, and controlled the decision to publish, as guarantor: LB.

Funding This study was funded by the Institute of Tropical Medicine's COVID-19 Pump Priming fund supported by the Flemish Government-Department of Economy, Science \& Innovation and by the Embassy of the UK in Belgium. LB is funded in part by the Research Foundation-Flanders (FWO) as part of her Senior Postdoctoral Fellowship. Research in Guinea was funded by the 4th framework agreement between the Institute of Tropical Medicine and Maferenyiah Research Centre funded by the Belgian Development Cooperation. The funders had no role in study design, data collection and analysis, decision to publish or preparation of the manuscript.

\section{Competing interests None declared.}

\section{Patient consent for publication Not applicable.}

Ethics approval This study involves human participants and was approved by We received ethical approval from the Institutional Review Board at the Institute of Tropical Medicine in Belgium (1372/20) and an approval for data collection from each participating hospital. Ethical permissions from all relevant country-level committees were also obtained, including from the Comité National d'éthique pour la Recherche en Santé in Guinea (058/CNERS/20); the Lagos University Teaching Hospital Health Research and Ethics Committee in Nigeria (LUTHHREC/EREV/0520/35); Muhimbili University of Health and Allied Sciences Senate Research and Publication Committee (MUHAS-REC-6-2020-282), Muhimbili National Hospital Ethics Committee (MNH/ IRB///2020/016), and National Institute for Medical Research (NIMPR/HQ/R.8a/ Vol.IX/3479) in Tanzania; and Makerere University School of Medicine Research Ethics Committee ((2020-150) and the Uganda National Council for Science and Technology (HS907ES) in Uganda. Participants gave informed consent to participate in the study before taking part.

Provenance and peer review Not commissioned; externally peer reviewed.

Data availability statement Data underlying this study can be made available upon reasonable request and data sharing agreements. These data include pseudonymised interview transcripts and aggregate monthly routine data indicators on caesarean section and labour induction. Data requests can be sent to the study PI Prof Lenka Benova at Ibenova@itg.be and the ethics committee at the Institute of Tropical Medicine at irb@itg.be. 
Supplemental material This content has been supplied by the author(s). It has not been vetted by BMJ Publishing Group Limited (BMJ) and may not have been peer-reviewed. Any opinions or recommendations discussed are solely those of the author(s) and are not endorsed by BMJ. BMJ disclaims all liability and responsibility arising from any reliance placed on the content. Where the content includes any translated material, BMJ does not warrant the accuracy and reliability of the translations (including but not limited to local regulations, clinical guidelines, terminology, drug names and drug dosages), and is not responsible for any error and/or omissions arising from translation and adaptation or otherwise.

Open access This is an open access article distributed in accordance with the Creative Commons Attribution Non Commercial (CC BY-NC 4.0) license, which permits others to distribute, remix, adapt, build upon this work non-commercially, and license their derivative works on different terms, provided the original work is properly cited, appropriate credit is given, any changes made indicated, and the use is non-commercial. See: http://creativecommons.org/licenses/by-nc/4.0/.

\section{ORCID iDs}

Aduragbemi Banke-Thomas http://orcid.org/0000-0002-4449-0131

Dinah Amongin http://orcid.org/0000-0002-1420-005X

Bosede Bukola Afolabi http://orcid.org/0000-0002-7511-7567

Alexandre Delamou http://orcid.org/0000-0002-9397-7106

\section{REFERENCES}

1 World Health Organization. WHO coronavirus disease (COVID-19) dashboard [online], 2020. Available: https://covid19.who.int/ [Accessed 22 Sep 2020].

2 Africa CDC Dashboard. Coronavirus disease 2019 (COVID-19) latest updated on the COVID-19 crises Africa CDC [online], 2020. Available: africacdc.org/covid-19/ [Accessed 1 Sep 2020].

3 WHO. Report of the WHO-China joint mission on coronavirus disease 2019 (COVID-19) [online], 2020. Available: https://www.who int/docs/default-source/coronaviruse/who-china-joint-mission-oncovid-19-final-report.pdf

4 Khan MSI, Nabeka H, Akbar SMF, et al. Risk of congenital birth defects during COVID-19 pandemic: draw attention to the physicians and policymakers. J Glob Health 2020;10.

5 Khalil A, Kalafat E, Benlioglu C, et al. SARS-CoV-2 infection in pregnancy: a systematic review and meta-analysis of clinical features and pregnancy outcomes. EClinicalMedicine 2020;25:100446

6 McClure EM, Kinney MV, Leisher SH, et al. Impact of COVID-19 on maternal and child health. Lancet Glob Health 2020;8:e1258.

7 Stein DT, Golub G, Rothschild CW. Bypassing high-quality maternity facilities: evidence from pregnant women in peri-urban Nairobi. Health Policy and Planning 2020:czaa092.

8 Chmielewska B, Barratt I, Townsend R, et al. Effects of the COVID-19 pandemic on maternal and perinatal outcomes: a systematic review and meta-analysis. Lancet Glob Health 2021;9:e759-72. doi:10.1016/S2214-109X(21)00079-6

9 Semaan A, Audet C, Huysmans E, et al. Voices from the frontline: findings from a thematic analysis of a rapid online global survey of maternal and newborn health professionals facing the COVID-19 pandemic. BMJ Glob Health 2020;5:e002967.

10 Kotlar B, Gerson E, Petrillo S, et al. The impact of the COVID-19 pandemic on maternal and perinatal health: a scoping review. Reprod Health 2021;18. doi:10.1186/s12978-021-01070-6

11 Kruk ME, Hermosilla S, Larson E, et al. Bypassing primary care clinics for childbirth: a cross-sectional study in the Pwani region, United Republic of Tanzania. Bull World Health Organ 2014:92:246-53.

12 Ashish KC, Peterson SS, Gurung R, et al. The perfect storm: disruptions to institutional delivery care arising from the COVID-19 pandemic in Nepal. J Glob Health 2021;11:05010. doi:10.7189/ jogh.11.05010

13 Shapira G, Ahmed T, Drouard SHP, et al. Disruptions in maternal and child health service utilization during COVID-19: analysis from eight
sub-Saharan African countries. Health Policy Plan 2021;36:czab064 doi:10.1093/heapol/czab064

14 Pattinson R, Fawcus S, Gebhardt S, et al. The effect of the first wave of Covid-19 on use of maternal and reproductive health services and maternal deaths in South Africa. O \& G Forum 2020;30:36-44.

15 Goyal M, Singh P, Singh K, et al. The effect of the COVID-19 pandemic on maternal health due to delay in seeking health care: experience from a tertiary center. Int $J$ Gynaecol Obstet 2021;152:231-5. doi:10.1002/ijgo.13457

16 Wanyana D, Wong R, Hakizimana D. Rapid assessment on the utilization of maternal and child health services during COVID-19 in Rwanda. Public Health Action 2021;11:12-21.

17 Zimmerman LA, Desta S, Karp C, et al. Effect of the COVID-19 pandemic on health facility delivery in Ethiopia; results from PMA Ethiopia's longitudinal panel. PLOS Glob Public Health 2021;1:e0000023. doi:10.1371/journal.pgph.0000023

18 Creswell JW, Clark VL. Choosing a mixed method design. In: Designing and conducting mixed methods research. 3rd edn. SAGE Publications Inc, 2011: 53-107.

19 Hale T, Angrist N, Goldszmidt R, et al. A global panel database of pandemic policies (Oxford COVID-19 government response Tracker). Nat Hum Behav 2021;5:529-38. doi:10.1038/s41562-021-01079-8

20 Salyer SJ, Maeda J, Sembuche S, et al. The first and second waves of the COVID-19 pandemic in Africa: a cross-sectional study. Lancet 2021;397:1265-75. doi:10.1016/S01406736(21)00632-2

21 Gale NK, Heath G, Cameron E, et al. Using the framework method for the analysis of qualitative data in multi-disciplinary health research. BMC Med Res Methodol 2013;13:117.

22 Haider N, Osman AY, Gadzekpo A, et al. Lockdown measures in response to COVID-19 in nine sub-Saharan African countries. BMJ Glob Health 2020;5:e003319. doi:10.1136/bmjgh-2020-003319

23 Banke-Thomas A, Makwe CC, Balogun M, et al. Utilization cost of maternity services for childbirth among pregnant women with coronavirus disease 2019 in Nigeria's epicenter. Int J Gynecol Obstet 2021;152:242-8.

24 Banke-Thomas A, Ayomoh FI, Abejirinde I-OO, et al. Cost of utilising maternal health services in low- and middle-income countries: a systematic review. IntJ Health Policy Manage 2021;10

25 Kumari V, Mehta K, Choudhary R. COVID-19 outbreak and decreased hospitalisation of pregnant women in labour. Lancet Glob Health 2020;8:e1116-7.

26 Bonnet E, Bodson O, Le Marcis F, et al. The COVID-19 pandemic in francophone West Africa: from the first cases to responses in seven countries. BMC Public Health 2021;21:1490.

27 Sachs JD, Abdool Karim S, Aknin L, et al. Lancet COVID-19 commission statement on the occasion of the 75th session of the UN General assembly. Lancet 2020;396:1102-24. doi:10.1016/ S0140-6736(20)31927-9

28 Mortazavi F, Mehrabadi M, KiaeeTabar R. Pregnant women's well-being and worry during the COVID-19 pandemic: a cross-sectional study. BMC Pregnancy Childbirth 2021;21:59. doi:10.1186/s12884-02103548-4

29 Galle A, Semaan A, Huysmans E, et al. A double-edged swordtelemedicine for maternal care during COVID-19: findings from a global mixed-methods study of healthcare providers. BMJ Glob Health 2021;6:e004575. doi:10.1136/bmjgh-2020-004575

30 Semaan A, Banke-Thomas A, Pembe A. "We are not going to shut down, because we cannot postpone pregnancy" - A mixed-methods study of the provision of maternal healthcare in six referral maternity wards in four sub-Saharan African countries during the COVID-19 pandemic. BMJ Global Health 2021.

31 Yerger $\mathrm{P}$, Jalloh $\mathrm{M}$, Coltart CEM, et al. Barriers to maternal health services during the Ebola outbreak in three West African countries: a literature review. BMJ Glob Health 2020;5:e002974. doi:10.1136/ bmjgh-2020-002974

32 Balogun M, Banke-Thomas A, Sekoni A, et al. Challenges in access and satisfaction with reproductive, maternal, newborn and child health services in Nigeria during the COVID-19 pandemic: a cross-sectional survey. PLoS One;16:e0251382. doi:10.1371/journal.pone.0251382 